\title{
Microemulsion Using Polyoxyethylene Sorbitan Trioleate and Its Usage for Skin Delivery of Resveratrol to Protect Skin against UV-Induced Damage
}

\author{
Reiko Yutani,* Reiko Teraoka, and Shuji Kitagawa \\ Kobe Pharmaceutical University; 4-19-1 Motoyamakita-machi, Higashinada-ku, Kobe 658-8558, Japan. \\ Received April 30, 2015; accepted June 27, 2015
}

We examined the phase behavior of various polyoxyethylene sorbitan fatty acid ester (polysorbates)/ ethanol/isopropyl myristate (IPM)/150 m M NaCl solution ( $\mathrm{NaClaq)}$ systems in order to prepare a microemulsion containing a low ratio of ethanol, which is more suitable for in vivo application. Using polyoxyethylene sorbitan trioleate (Tween 85 ), which has a large lipophilic moiety, as a surfactant component, single-phase domain of the phase diagram was the largest of all the polysorbates examined, and in particular a large oilrich single-phase domain was obtained. When the ratio of Tween 85 to ethanol was changed from $1: 1$ to $3: 1$, the oil-rich single-phase domain further expanded, which led to a reduced ethanol concentration in the preparation. Thus, we determined the composition of the microemulsion to be Tween $85:$ ethanol:IPM:NaClaq= $30: 10: 53: 7$, and used it for skin delivery of resveratrol. Microemulsion gel was also prepared by adding $6.5 \%$ Aerosil $^{\circledR} 200$ into the microemulsion for ease of topical application. When applied with each vehicle, delivery of resveratrol into guinea pig skin in vitro was significantly enhanced compared with that by IPM, and resveratrol incorporated into the skin by microemulsion gel decreased lipid peroxidation to $29.5 \%$ compared with that of the control. Pretreatment of guinea pig dorsal skin with the microemulsion gel containing resveratrol almost completely prevented UV-B-induced erythema formation in vivo. These findings demonstrate that the microemulsion using Tween 85 containing a minimal concentration of ethanol enhanced the skin delivery of resveratrol and the incorporated resveratrol exhibited a protective effect against UV-induced oxidative damage.

Key words resveratrol; microemulsion; polyoxyethylene sorbitan trioleate; skin delivery; photoprotection; phase diagram

Resveratrol (trans-3,5,4'-trihydroxystilbene, Fig. 1) is a naturally occurring polyphenolic phytoalexin synthesized by a wide variety of plant species, including grapes, berries and peanuts, in response to environmental stress and microbial infection. $^{1,2)}$ It has been suggested in several studies that resveratrol is a potent antioxidant with anti-inflammatory and antiproliferative properties ${ }^{1-5}$; thus, the application of resveratrol for topical purposes has been examined. However, since the penetration of resveratrol into skin is limited, ${ }^{6)}$ the use of an enhancement system is an effective way of facilitating the penetration and the exertion of its physiological activities in skin.

To improve the skin delivery of resveratrol, we have used microemulsion as an enhancement system. ${ }^{6)}$ Microemulsions consist of an aqueous phase, an oil phase, a surfactant and a co-surfactant component, which are thermodynamically stable and have been shown to have high solubilization capacity and to facilitate the skin incorporation of both

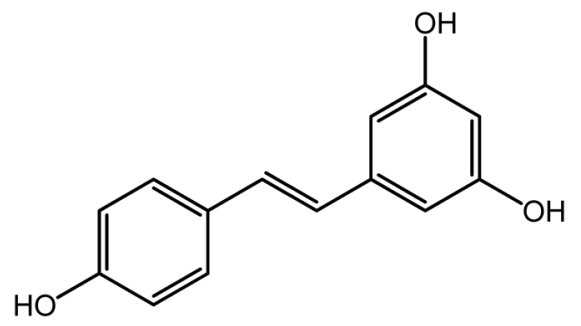

Fig. 1. Chemical Structure of Resveratrol hydrophilic and lipophilic drugs. ${ }^{7,8)}$ In previous studies, we revealed that a microemulsion consisting of polyoxyethylene sorbitan monooleate (Tween 80), ethanol, isopropyl mysistate (IPM) and $150 \mathrm{~mm} \mathrm{NaCl}$ solution (NaClaq) at a weight ratio of $30: 30: 33: 7$ improved the intradermal delivery of polyphenols, such as quercetin, ${ }^{9)}$ genistein ${ }^{10)}$ and chlorogenic acid, ${ }^{11)}$ and the polyphenols incorporated into skin prevented lipid peroxidation $^{9,10)}$ and UV-induced erythema formation. ${ }^{10,11)}$ This microemulsion also increased the incorporation of resveratrol into skin, compared with that by IPM. ${ }^{6}$

In this study, we first attempted to prepare a microemulsion containing a lower ratio of ethanol, which is more suitable for in vivo application to avoid skin irritancy. Pseudo-ternary phase diagrams using various polyoxyethylene sorbitan fatty acid esters (polysorbates) were constructed and the composition of the microemulsion was determined. Microemulsion gel was also prepared using Aerosil ${ }^{\circledR} 200$ as a gelling agent, to retain the preparation at the site of application for practical use. Next, intradermal and transdermal delivery of resveratrol was examined in vitro using guinea pig skin, and the antilipoperoxidative activity of resveratrol incorporated by microemulsion gel was assessed by the thiobarbituric acid (TBA) test. Furthermore, the effect of resveratrol incorporated by microemulsion gel on UV-B-induced erythema formation was examined in vivo using guinea pigs.

\section{Results and Discussion}

We first examined the phase behavior of various polysorbate/ethanol/IPM/NaClaq systems. Pseudo-ternary phase dia- 
(a)

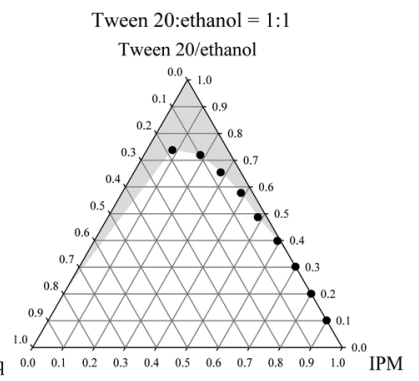

(c)

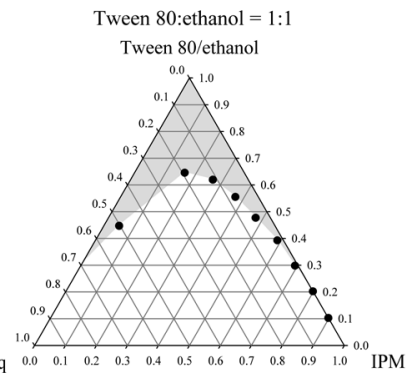

(e)

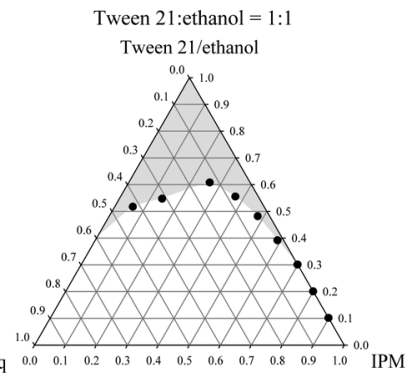

(g)

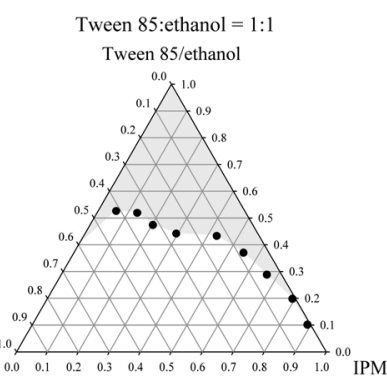

(i) Tween 85 : ethanol $=3: 1$ Tween $85 /$ ethanol

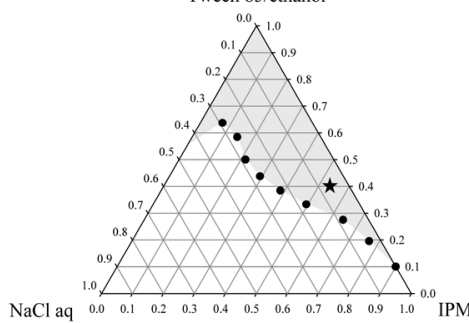

(b)

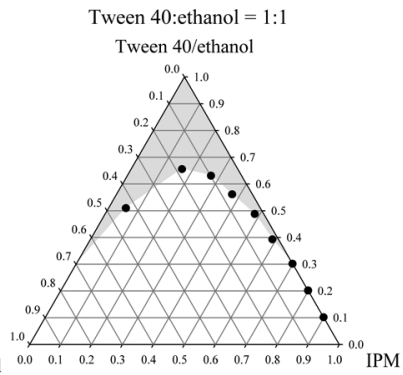

(d)

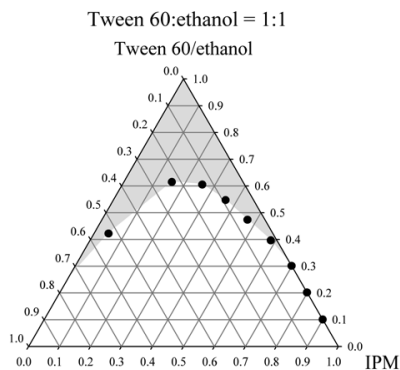

(f)

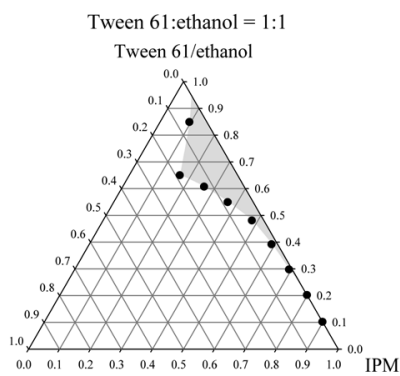

(h)

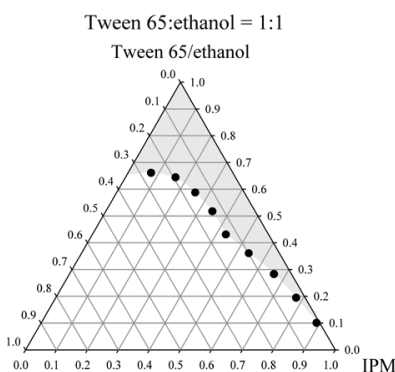

Fig. 2. Pseudo-ternary Phase Diagrams at the Weight Ratio of Polysorbate to Ethanol, $1: 1$ for Tween 20 (a), Tween 40 (b), Tween 80 (c), Tween 60 (d), Tween 21 (e), Tween 61 (f), Tween 85 (g) and Tween 65 (h), and 3:1 for Tween 85 (i)

Single-phase domain is shown in gray in each picture. The composition of Tween 85 microemulsion used in this study is depicted with a star in Fig. 2(i).

grams using different polysorbates as a surfactant component are presented in Fig. 2. The upper part of the plots shown in gray on the diagrams represents the single-phase domain. As shown in Figs. 2(a)-(d), the single-phase domain slightly expanded with increasing hydrophobicity of the fatty acid chain (polyoxyethylene (20) sorbitan monolaurate (Tween 20)<poly- 
(a)

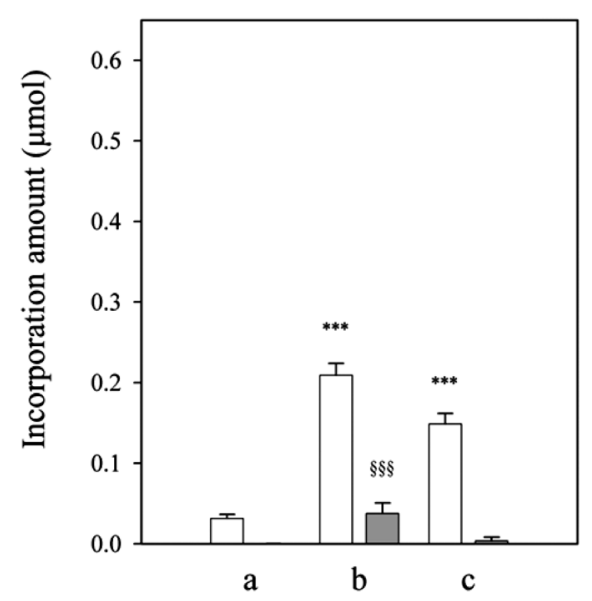

(b)

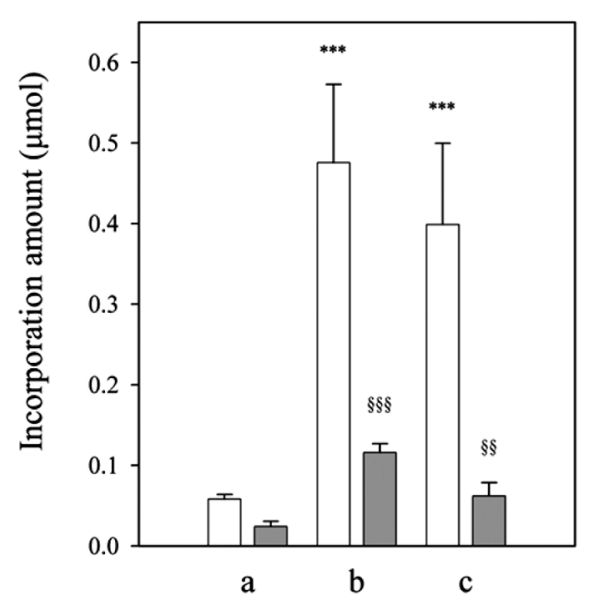

Fig. 3. Amount of Incorporation of Resveratrol into the Skin and into the Receptor Compartment When Applied with Each Vehicle to Guinea Pig Dorsal Skin for $6 \mathrm{~h}$ (a) and $20 \mathrm{~h}$ (b): a, IPM; b, Tween 85 Microemulsion; c, Microemulsion Gel

The open bars represent the amount of resveratrol incorporated into the skin and the shaded bars represent the amount of resveratrol that permeated into the receptor compartment. Data are mean \pm S.D. of at least 3 values. ${ }^{* * *} p<0.001$, compared with the data in the skin of IPM; ${ }^{\S \S} p<0.001$, ${ }^{\S \S} p<0.01$, compared with the data in the receptor compartment of IPM.

oxyethylene (20) sorbitan monopalmitate (Tween 40) $<$ Tween $80<$ polyoxyethylene (20) sorbitan monostearate (Tween 60)). The phase behaviors of polyoxyethylene (4) sorbitan monolaurate (Tween 21) and polyoxyethylene (4) sorbitan monostearate (Tween 61), which have smaller hydrophilic groups, differed from each other. As for Tween 21, the single-phase domain was larger than for Tween 20, which has the same lipophilic group, as shown in Figs. 2(a) and (e); as for Tween 61, on the other hand, the single-phase domain was smaller than for Tween 60, as shown in Figs. 2(d) and (f). Polyoxyethylene (20) sorbitan trioleate (Tween 85) and polyoxyethylene (20) sorbitan tristearate (Tween 65), as shown in Figs. 2(g) and (h), which have larger lipophilic moieties, exhibited a larger oil-rich single-phase domain than Tween 80 and Tween 60, respectively, as shown in Figs. 2(c) and (d). Tween 85 displayed the largest single-phase domain of all the polysorbates examined, possibly because its large sizes of both hydrophilic and lipophilic moieties enabled the incorporation of greater amounts of oil and water. In addition, the oil-rich single-phase domain further expanded upon changing the ratio of Tween 85 to ethanol from $1: 1$ to $3: 1$, as shown in Figs. 2(g) and (i). For decreasing the ratio of ethanol in a preparation, we thus determined the composition of the microemulsion to be Tween 85 : ethanol:IPM: NaClaq $=30: 10: 53: 7$ (Tween 85 microemulsion, depicted with a star in Fig. 2(i)), by modifying the composition of the microemulsion using Tween 80 (Tween 80 : ethanol: IPM $: \mathrm{NaClaq}=30: 30: 33: 7) .{ }^{6,9-11)}$

We next examined the intradermal and transdermal delivery of resveratrol to assess the drug delivery potential of Tween 85 microemulsion. Microemulsion gel was also prepared by adding 6.5\% Aerosil ${ }^{\circledR} 200$ into Tween 85 microemulsion for the ease of topical application. As shown in Fig. 3, both Tween 85 microemulsion and microemulsion gel significantly improved the skin delivery of resveratrol at both time points compared with that by IPM. The amounts of incorporation in skin by the microemulsion and that by microemulsion gel after $20 \mathrm{~h}$ of application were about 8 -fold and 7-fold higher than that by IPM, respectively. Permeation of resveratrol into the receptor compartment was found for all vehicles, but the amount of permeation was smaller than the amount of incorporation in skin.

To confirm the antioxidative effect of resveratrol in skin, we then examined the antilipoperoxidative activity of resveratrol incorporated into skin by microemulsion gel. In terms of the results, the incorporated resveratrol significantly inhibited lipid peroxidation: the production of TBA-reactive substances decreased to $29.5 \%$ compared with that for the control gel without resveratrol.

To clarify the effects of topical application of resveratrol with the microemulsion gel on UV-induced damage, we examined the effects of resveratrol incorporated on UV-B-induced erythema formation using guinea pigs. As shown in Fig. 4(b), skin erythema was observed on the left side of the back of the guinea pig where microemulsion gel without resveratrol had been preloaded. On the other hand, erythema formation was significantly and almost completely inhibited on the right side where microemulsion gel with resveratrol had been preloaded. As shown in Fig. 4(c), the increase of skin redness measured with a color reader at the resveratrol-preloaded site was also prevented compared with that at the control site.

The present study demonstrated that Tween 85 microemulsion that contained a low ratio of ethanol significantly improved the skin delivery of resveratrol. About $0.23 \%$ of the resveratrol added to the donor compartment was incorporated into the skin, which is slightly higher than the previously reported value $(0.15 \%$ for microemulsion using Tween 80$){ }^{6}$ ) These enhancing effects of polysorbate microemulsions are probably attributable to the high solubilization capacity of polysorbates, which are assumed to form large interfacial surfactant film between inner and outer phases in the microemulsion, enabling the incorporation of greater amounts of drug molecules. ${ }^{7,8)}$ Since the increased solubility in the vehicle does not necessarily lead to increased skin delivery of the drug, microemulsions seem to increase the drug diffusion process and the partition into the stratum corneum. ${ }^{11)}$ High drug mobility in the vehicle accelerating drug access to the skin surface and the effect of microemulsion components themselves as penetration enhancers seem to be involved in the mechanism of 
(a)

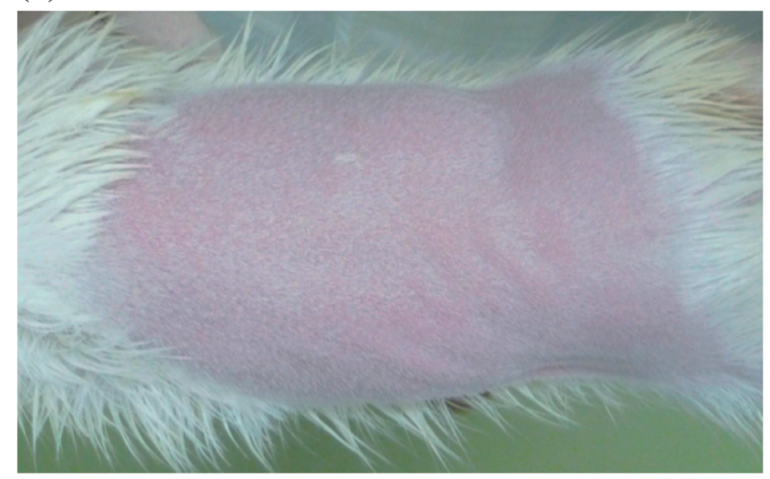

(b)

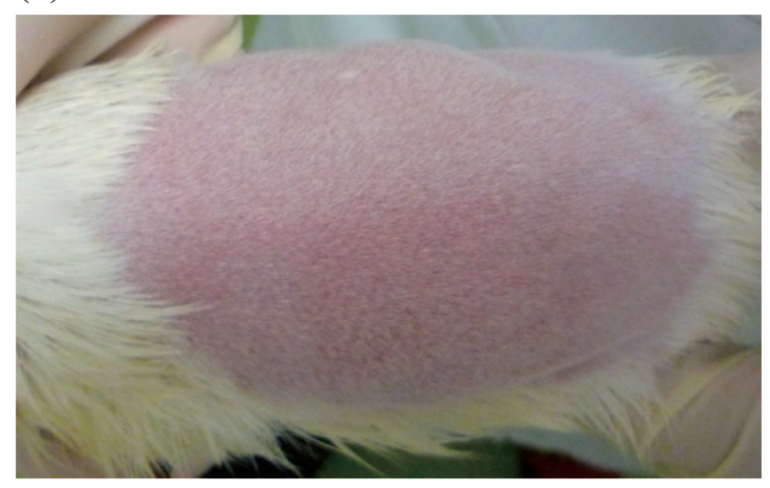

(c)

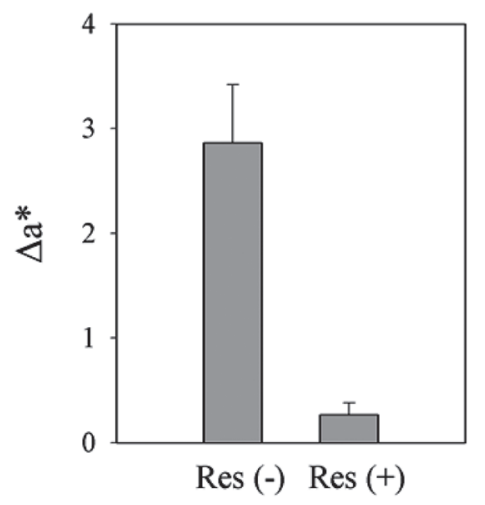

Fig. 4. Effect of Resveratrol Incorporated into Skin by Microemulsion Gel on UV-B-Induced Erythema Formation

(a) and (b) represent photographs of the back of a guinea pig before UV irradiation and after UV irradiation, respectively. Microemulsion gel without resveratro was applied to the left side (control site, which is shown in the lower part of each picture) of the guinea pig dorsal skin and microemulsion gel with $200 \mu \mathrm{mol}$ resveratrol was applied to the right side (resveratrol-preloaded site). (c) represents the difference of $a^{*}$ values between before and after irradiation $\left(\Delta a^{*}\right)$ : Res $(-)$, contro site; Res $(+)$, resveratrol-preloaded site. Data are mean \pm S.D. of 3 values. Measurements were performed at 3 points at each site.

enhancement by microemulsions. ${ }^{7,8)}$

UV-B, which is an essential component of sunlight, can cross the whole epidermis layer and penetrate the upper dermis, resulting in erythema, edema, sunburn cells, hyperplasia, immunosuppression, photoaging and photocarcinogenesis, which are directly or indirectly related to the excessive production of reactive oxygen species. ${ }^{1,3,12)}$ It has been demonstrated that photo-damage can be reduced when the skin is supplemented with antioxidants. ${ }^{3,12)}$ In this study, resveratrol incorporated into skin by microemulsion gel containing a low ratio of ethanol effectively prevented against UV-B-induced erythema formation, as revealed for genistein and chlorogenic acid in previous studies. ${ }^{10,11)}$ This effect of resveratrol is attributable to its strong photoprotective properties, for example, the reduction of reactive oxygen species, the inhibition of cyclooxygenase and the decreased infiltration of leukocytes. . $3,3,4,12,13)$

\section{Conclusion}

The results obtained from the phase diagrams demonstrated that Tween 85, which has a large lipophilic moiety, exhibited a large oil-rich single-phase domain. We thus chose Tween 85 as a surfactant to prepare a microemulsion containing a low ratio of ethanol, and confirmed the photoprotective effect of resveratrol incorporated into skin by the microemulsion gel. The findings obtained in this study demonstrated that Tween 85 microemulsion would be useful for enhancement of the skin delivery of resveratrol to protect the skin against UV-induced oxidative damage.

\section{Experimental}

Materials Resveratrol and trans-ferulic acid were purchased from Tokyo Chemical Industry Co., Ltd. (Tokyo, Japan). Tween 20, Tween 40, Tween 80, Tween 60, Tween 85, IPM, ethanol and 2-thiobarbituric acid were purchased from Nacalai Tesque, Inc. (Kyoto, Japan). Tween 21, Tween 61 and Tween 65 were kindly provided by Croda Japan KK (Tokyo, Japan). Aerosil ${ }^{\circledR} 200$ was purchased by Nippon Aerosil Co., Ltd. (Tokyo, Japan). All other reagents were purchased from Wako Pure Chemical Industries, Ltd. (Osaka, Japan). Dorsal skin excised from Hartley male guinea pigs, whose fur on the back had been cut using hair clippers beforehand, was purchased from Japan SLC, Inc. (Hamamatsu, Japan), and Hartley male guinea pigs were also obtained.

Pseudo-ternary Phase Diagram Construction Pseudoternary phase diagrams were constructed at room temperature. NaClaq was used as an aqueous phase, IPM as an oil phase, one of the polysorbates (Tween 20, Tween 40, Tween 80, Tween 60, Tween 21, Tween 61, Tween 85 and Tween 65 ) as a surfactant component and ethanol as a co-surfactant component. The surfactant and co-surfactant were mixed at a weight ratio of $1: 1$ for all polysorbates and $3: 1$ for Tween 85 . At each ratio, $1 \mathrm{~g}$ of surfactant/co-surfactant/oil phase mixture was prepared at weight ratios from 1:9 to 9:1 ((surfactant/cosurfactant): oil phase), and then titrated with $10 \mu \mathrm{L}$ of aqueous solution and mixed using a vortex mixer. This procedure was repeated until the transparent dispersion became translucent or turbid and the weight ratio at this time was plotted on the phase diagrams. To estimate the whole single-phase domain, surfactant/co-surfactant/aqueous phase mixtures were also prepared, and the boundary of the single-phase domain was determined.

Preparation of Microemulsion and Microemulsion Gel Tween 85 microemulsion was obtained by vortex mixing and resveratrol was added to it at a concentration of $200 \mathrm{~mm}$ under solubilized conditions. Microemulsion gel was prepared by adding $6.5 \%$ Aerosil $^{\circledR} 200$ as a gelling agent into the preformed microemulsion and mixing by magnetic stirring for about $1 \mathrm{~h}$.

In Vitro Study on Skin Delivery of Resveratrol In vitro 
studies on the intradermal and transdermal delivery of resveratrol were performed as described previously, ${ }^{6)}$ using guinea pig dorsal skin mounted in a Franz-type diffusion cell with a water jacket $\left(37^{\circ} \mathrm{C}\right)$. The available diffusion area was approximately $0.83 \mathrm{~cm}^{2}$ and the receptor cell had a volume of about $4.6 \mathrm{~mL}$. After $2 \mathrm{~h}$ of pretreatment of the skin with NaClaq, both donor and receptor compartments were washed and $1 \mathrm{~mL}$ of vehicle containing $200 \mu \mathrm{mol}$ resveratrol was added to the donor compartment. IPM suspending resveratrol was used as a control. Phosphate-buffered saline (PBS, $\mathrm{pH}$ 7.4) was added to the receptor compartment. After 6 or $20 \mathrm{~h}$ of treatments, the skin was removed from the cell, and the treated skin area was punched out and washed with ice-cold methanol. Each skin sample was minced and placed in $10 \mathrm{~mL}$ of methanol, and then homogenized using a tissue homogenizer, Polytron PT3100 (Kinematica AG, Lucerne, Switzerland), at $5000 \mathrm{rpm}$ for $1 \mathrm{~min}$. The samples were then centrifuged and the supernatant layer was used for determination by HPLC. The receptor solution was also collected after treatment to determine the amount of resveratrol that had permeated through the skin by HPLC.

Lipid Peroxidation Assay The anti-lipoperoxidative activity of resveratrol incorporated into skin by microemulsion gel was evaluated in vitro by the formation of malondialdehyde (MDA), as described previously. ${ }^{9,10)}$ After $20 \mathrm{~h}$ of treatment of microemulsion gel containing $200 \mu \mathrm{mol}$ resveratrol with skin as described above, the skin was homogenized in $1.15 \% \mathrm{KCl}$ solution. Ammonium iron(II) sulfate and sodium citrate were added to $1.0 \mathrm{~mL}$ of sample solution containing about $0.2 \mathrm{mg}$ of protein to final concentrations of $50 \mu \mathrm{m}$ and $2 \mathrm{~mm}$, respectively, and the solution was kept for $30 \mathrm{~min}$ at $37^{\circ} \mathrm{C}$. Then, $0.20 \mathrm{~mL}$ of $8.1 \%$ sodium dodecyl sulfate solution, $1.5 \mathrm{~mL}$ of acetate buffer adjusted to $\mathrm{pH} 3.5,50 \mu \mathrm{L}$ of $0.8 \%$ butylhydroxytoluene in glacial acetic acid, $1.5 \mathrm{~mL}$ of $0.8 \%$ TBA solution, and $0.7 \mathrm{~mL}$ of $5 \mathrm{~mm}$ iron(III) chloride solution were added in this order, followed by $60 \mathrm{~min}$ of incubation at $60^{\circ} \mathrm{C}$. After cooling, MDA-TBA complex was extracted with $5 \mathrm{~mL}$ of $n$-butanol and pyridine mixed solution, centrifuged at $1670 \times \mathbf{g}$ for $10 \mathrm{~min}$, and the absorbance of the supernatants was measured at $532 \mathrm{~nm}$.

Observation of Erythema Formation Erythema formation induced by UV irradiation was observed as described previously, ${ }^{10,11)}$ following the protocol approved by the Animal Experimentation Committee of Kobe Pharmaceutical University. Fur on the dorsal skin of guinea pigs was cut using hair clippers and shavers and dirt on the skin was washed off. Approximately $24 \mathrm{~h}$ later, microemulsion gel containing $200 \mu \mathrm{mol}$ resveratrol was applied to the right side of the dorsal skin, while microemulsion gel without resveratrol was applied to the left side. After approximately $24 \mathrm{~h}$, microemulsion gel remaining on the skin surface was wiped off with wet gauze repeatedly. After drying the skin surface for about $5 \mathrm{~h}$, both sides of the skin were irradiated for $24 \mathrm{~min}$ using UV light at an intensity of $138 \mu \mathrm{W} / \mathrm{cm}^{2}$ measured with a UV-Meter 340 (Ando Keiki Co., Ltd., Tokyo, Japan). UV-B lamps, GL20SE (Sankyo Denki Co., Ltd., Kanagawa, Japan), emitting a continuous light spectrum between 280 and $380 \mathrm{~nm}$ with peak emission at $306 \mathrm{~nm}$, were used. Erythema formation was evaluated from photographs of the back of the guinea pigs as well as measurement of the skin redness $\left(a^{*}\right)$ with a color reader, CR-13 (Konica Minolta, Inc., Tokyo, Japan).

Statistical Analysis The results are expressed as mean \pm standard deviation (S.D.) of at least 3 values. Data were analyzed by one-way ANOVA followed by Dunnett's test. Differences were regarded as significant at $p<0.05$.

Conflict of Interest The authors declare no conflict of interest.

\section{References}

1) Caddeo C., Teskač K., Sinico C., Kristl J., Int. J. Pharm., 363, 183-191 (2008).

2) Udenigwe C. C., Ramprasath V. R., Aluko R. E., Jones P. J. H., Nutr. Rev., 66, 445-454 (2008).

3) Afaq F., Mukhtar H., Exp. Dermatol., 15, 678-684 (2006).

4) Bisht K., Wagner K. H., Bulmer A. C., Toxicology, 278, 88-100 (2010).

5) Jang M., Cai L., Udeani G. O., Slowing K. V., Thomas C. F., Beecher C. W. W., Fong H. H. S., Farnsworth N. R., Kinghorn A. D., Mehta R. G., Moon R. C., Pezzuto J. M., Science, 275, 218-220 (1997).

6) Yutani R., Morita S. Y., Teraoka R., Kitagawa S., Chem. Pharm. Bull., 60, 989-994 (2012).

7) Kreilgaard M., Adv. Drug Deliv. Rev., 54 (Suppl. 1), S77-S98 (2002).

8) Pappinen S., Urtti A., "Percutaneous Penetration Enhancers," 2nd ed., ed. by Smith E. W., Maibach H. I., CRC, Boca Raton, 2006, pp. 109-116.

9) Kitagawa S., Tanaka Y., Tanaka M., Endo K., Yoshii A., J. Pharm. Pharmacol., 61, 855-860 (2009).

10) Kitagawa S., Inoue K., Teraoka R., Morita S. Y., Chem. Pharm. Bull., 58, 398-401 (2010).

11) Kitagawa S., Yoshii K., Morita S. Y., Teraoka R., Chem. Pharm. Bull., 59, 793-796 (2011).

12) Nichols J. A., Katiyar S. K., Arch. Dermatol. Res., 302, 71-83 (2010).

13) Park K., Lee J. H., Oncol. Rep., 19, 413-417 (2008). 